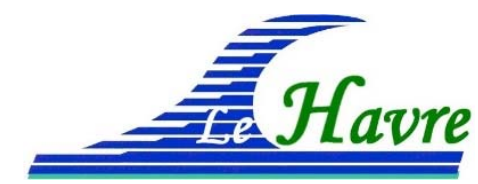

XVI İmes Journées Nationales Génie Côtier - Génie Civil

Le Havre, 2020

DOI:10.5150/jngcgc.2020.046 C Editions Paralia CFL

disponible en ligne - http://www.paralia.fr - available online

\title{
Propositions de conceptions évolutives des marinas du futur, en tenant compte de l'élévation prévisible du niveau des mers
}

\author{
Jean-Marc BEYNET ${ }^{1}$, Jean-Pierre REDON ${ }^{2}$
}

1. Beynet-Consult, 35 rue de Peyrouse, 30320 Marguerittes, France. beynet.consult@gmail.com

2. Legal Consultant \& Partners, Plaza Universitat 3, Barcelona, 08007, Espagne. legalconsultant.partners@gmail.com

\section{Résumé :}

Les marinas réalisées en France depuis les années 1960-70 sont des aménagements portuaires qui seront difficilement adaptables pour prendre en compte l'élévation attendue du niveau des mers dans les décennies à venir, en raison du changement climatique. Selon le GIEC (2019), ce phénomène parait inéluctable et l'élévation prévisible pourrait varier dans de larges proportions en fonction des scenarios modélisés pour la réduction des rejets de $\mathrm{CO} 2$ dans l'atmosphère. En conséquence, il est souhaitable de proposer dorénavant des conceptions évolutives pour les marinas du futur, de manière à pouvoir anticiper l'élévation du niveau de l'eau, tout en maintenant l'amarrage du bateau de plaisance au plus près, et si possible devant l'habitation du propriétaire, ce qui est le principe même d'une marina. Dans la présente publication, ce sont de telles idées de conceptions évolutives, qui sont proposées pour les marinas du futur. Ces propositions prennent en considération le contexte réglementaire applicable en France métropolitaine, le respect de l'environnement, ainsi que la nécessité d'être autonome aussi bien pour l'assainissement que l'énergie. La conception évolutive des ouvrages de protection contre la houle des marinas est abordée également.

\section{Mots-clés :}

Marina, Habitat flottant, sur pilotis, Elévation niveau des mers, Conception évolutive, Contexte réglementaire, Energies renouvelables, Assainissement autonome

\section{Introduction}

En France, les premières marinas ont été réalisées sur le littoral méditerranéen dans les années 1960-70 (Port-Grimaud, Port-Camargue, Le Cap d'Agde, par exemple). Ce sont des ensembles immobiliers établis sur une concession d'endigage en combinant des habitations avec un port de plaisance. Les quais d'accostage des bateaux de plaisance sont disposés au plus près de la résidence de leur propriétaire. Ce concept était séduisant à l'époque. Mais de nos jours, les scientifiques, les climatologues, les politiques et les riverains ont pris conscience des risques de submersion marine pour ce type d'habitat en raison de l'élévation du niveau des mers dans les années à venir. Pour s'adapter, a priori, 


\section{Thème 4 - Ouvrages portuaires, offshore et de plaisance}

trois conceptions viennent à l'esprit : Habitat flottant et habitat sur pilotis, sur plan d'eau, ou en partie, sur berges ou talus.

\section{Habitat flottant}

Dans le cas de l'habitat flottant, figure 1, le quai d'accueil du bateau et l'habitation du propriétaire sont généralement regroupés sur un même ponton flottant, coulissant le long de pieux, permettant à l'ensemble (habitation et quai), de suivre les fluctuations du niveau d'eau (mer ou embouchure de fleuve ou graus de communication des lagunes avec la mer, par exemple).
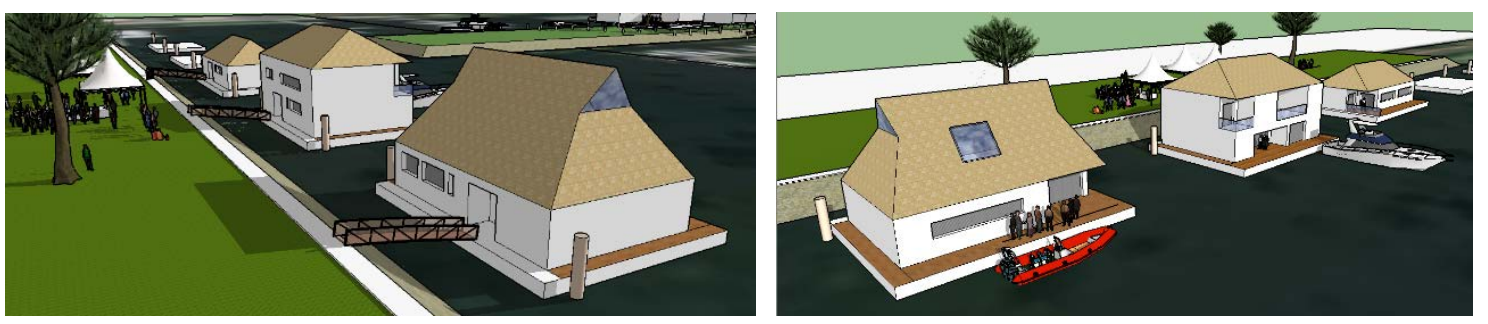

Figure 1. Habitat flottant (@ Beynet, 2019).

Mais une telle solution n'est pas optimale pour plusieurs raisons. Sur un plan d'eau abrité (étang, lac), elle peut s'envisager assez facilement. Par contre, s'il y a des risques d'agitation (vagues, clapots), cette solution ne pourra pas être mise en œuvre, sauf à prévoir un franc-bord important pour éviter que l'eau ne submerge le flotteur et pénètre dans l'habitation. Cette conception n'est pas idéale non plus si la profondeur d'eau est faible car dans ce cas, le flotteur (en fonction de son tirant d'eau) peut constituer un obstacle à la libre circulation des courants, ce qui parfois peut conduire à l'eutrophisation du plan d'eau. De plus, en faible profondeur l'ombre portée sur le fond peut être importante.

Sur le plan réglementaire, dès lors qu'ils se situent sur le domaine public ou privé des personnes publiques et qu'ils disposent d'équipements pouvant assurer leur mobilité ou non, les habitats flottants sont soumis à différents régimes d'autorisation. Les règles qui s'appliquent aux biens et aux droits, à caractère mobilier ou immobilier appartenant à des personnes publiques sont définies tant par le Code général de la propriété des personnes publiques (CG3P), que par le Code général des collectivités territoriales. En outre, les constructions et installations réalisées sur les dépendances domaniales peuvent également nécessiter une autorisation prévue par le Code de l'urbanisme et/ou par le Code de l'environnement.

Du point de vue strictement domanial, il convient de rappeler quelques principes fondamentaux applicables sur toute dépendance du domaine public, quelle que soit la personne publique propriétaire : 


\section{XVI'mes Journées Nationales Génie Côtier - Génie Civil \\ Le Havre 2020}

- L'obtention d'un titre d'occupation est requis avant toute installation sur le domaine public: l'article L. 2122-1 du CG3P indique : "Nul ne peut, sans disposer d'un titre l'y habilitant, occuper une dépendance du domaine public d'une personne publique [...] ou l'utiliser dans des limites dépassant le droit d'usage qui appartient à tous".

- une occupation du domaine public doit respecter l'affectation de la dépendance domaniale concernée à l'utilité publique. L'article L 2121-1 du CG3P dispose que : "Les biens du domaine public sont utilisés conformément à leur affectation à l'utilité publique. Aucun droit d'aucune nature ne peut être consenti s'il fait obstacle au respect de cette affectation".

- L'article L2124-1 du même code réaffirme ce principe protecteur de l'affectation à propos du domaine public maritime, en précisant notamment que les décisions d'utilisation du DPM tiennent compte de la vocation des zones concernées et des espaces terrestres avoisinants, et que tout changement substantiel d'utilisations de zones du DPM est soumis à enquête publique.

- Toute occupation ne peut qu'être temporaire (art. L. 2122-2 CG3P) et présente un caractère précaire et révocable (art. L. 2122-3 CG3P);

- Toute occupation entraîne la perception d'une redevance : l'article L2125-1 CG3P dispose que "Toute occupation ou utilisation du domaine public d'une personne publique mentionnée à l'article L. 1 donne lieu au paiement d'une redevance". Cette redevance est calculée sur la base d'une grille tarifaire et elle est due annuellement. Le paiement de la redevance est justifié par le bénéfice que l'utilisateur tire d'une occupation ou d'une utilisation "privative" du domaine et n'appelle, en contrepartie, aucun service de la part du gestionnaire du domaine.

L'étendue des droits attribués à l'occupant régulier du domaine public, varie selon qu'il se trouve sur le domaine de l'Etat, ou celui d'une collectivité territoriale. Lorsqu'il s'agit d'une dépendance domaniale de l'Etat, l'occupant peut disposer d'un droit qui lui confère les prérogatives et les obligations du propriétaire pendant la durée de l'autorisation (Art. L.2122-6 du CG3P).

L'occupant d'une dépendance du domaine d'une collectivité territoriale est dans une situation différente. Le Code général des collectivités territoriales (CGCT) reconnait que le titre d'occupation puisse conférer de manière expresse des droits réels, mais cette possibilité est restreinte à des activités ayant en vue "l'accomplissement, pour le compte de la collectivité qui l'accorde, d'une mission de service public ou d'une opération d'intérêt général relevant de sa compétence" (Article L1311-5 CGCT). Ce texte ne reconnaît comme satisfaisant à la condition d'intérêt public, que "(...) les activités ayant trait à l'exploitation du port ou de l'aéroport ou qui sont de nature à contribuer à leur animation ou à leur développement". Ce dernier point conduit à préciser que dans le cas d'une activité économique sur le domaine public, l'article L2122-1-1 du CG3P exige que l'attribution fasse l'objet d'une procédure de sélection librement organisée par l'autorité 


\section{Thème 4 - Ouvrages portuaires, offshore et de plaisance}

compétente, et présentant toutes les garanties d'impartialité et de transparence, après procédure de publicité permettant aux candidats potentiels de se manifester.

Par ailleurs, s'il ressort de la jurisprudence qu'un logement flottant sur une dépendance $\mathrm{du}$ domaine public ne peut en aucun cas être vendu avec son emplacement du fait de l'inaliénabilité du domaine public (Conseil d'Etat, 01/03/1989, Béro, n 71140 : pour une péniche transformée en logement). Cependant, la Haute juridiction permet le transfert de l'autorisation d'occupation du domaine public, avec l'accord écrit du gestionnaire du domaine public (CE 18/08/2015 req. $\mathrm{n}^{\circ} 387315$ ).

Pour le domaine public fluvial, le gestionnaire du DPF peut délimiter des zones d'accueil réservées à cette utilisation particulière (Art. L2124-13 du CG3P). Les maisons flottantes sont donc regroupées dans des zones de stationnement pouvant être gérées sous forme de concessions, ou en régie directe par le gestionnaire du domaine.

Pour le DPM, la règle -de caractère général- est celle énoncée par l'article L2124-1 CG3P évoqué plus haut ; c'est-à-dire que toute décision d'utilisation du domaine public maritime doit tenir compte de la vocation des zones concernées et de celles des espaces terrestres avoisinants, ainsi que des impératifs de préservation des sites et paysages du littoral et des ressources biologiques.

S'agissant particulièrement des plages, cette vocation a d'ailleurs été consacrée par l'article 30 de la loi "Littoral" du 3 janvier 1986, dont les termes figurent aujourd'hui à l'alinéa 2 de l'article L321-9 du code de l'environnement: "l'usage libre et gratuit des plages constitue leur destination fondamentale au même titre que leur affectation aux cultures marines".

Sur le domaine portuaire, la marge de manœuvre de l'auteur du document local d'urbanisme est très étroite: si le port existe, il ne peut que se conformer à la vocation des dépendances du port. Ce n'est que dans l'hypothèse où des terrains situés dans les limites administratives du port n'auraient pas été affectés au service public portuaire qu'il peut être envisagé d'installer des maisons flottantes ou sur pilotis. Dans ce cas il faudra encore s'assurer de la compatibilité d'un tel aménagement avec le document d'urbanisme opposable.

Un point important: sans équipements de mobilité, tout habitat flottant sera réputé immobilisé à perpétuelle demeure, et de ce fait soumis au régime de droit commun. Indifféremment au fait que l'habitat flottant se trouve ou non sur le domaine public, la jurisprudence indique que les maisons flottantes peuvent constituer des constructions au sens de l'article L421-1 du code de l'urbanisme. Elles sont donc soumises au respect des règles d'urbanisme et notamment à celles relatives aux autorisations de construire. 


\section{XVİ̀mes Journées Nationales Génie Côtier - Génie Civil \\ Le Havre 2020}

\section{Habitat sur pilotis}

\subsection{Pilotis sur plan d'eau}

Cette conception est séduisante, car tout en maintenant l'amarrage du bateau au plus près de l'habitation du propriétaire, les fonctions sont dissociées : le plancher de l'habitat est calé au niveau le plus haut raisonnable en fonction de l'élévation prévisible du niveau de la mer, en prenant même une marge de sécurité, alors que le quai est fixé au contraire plus bas sur l'eau, pour permettre le transfert aisé des plaisanciers entre le bateau et le quai, figure 2. Et ce quai bas, peut facilement être surélevé ensuite, en fonction de l'élévation du niveau de la mer. Dans certains cas, si l'agitation reste modérée, ce quai fixe pourrait être remplacé par un ponton flottant.

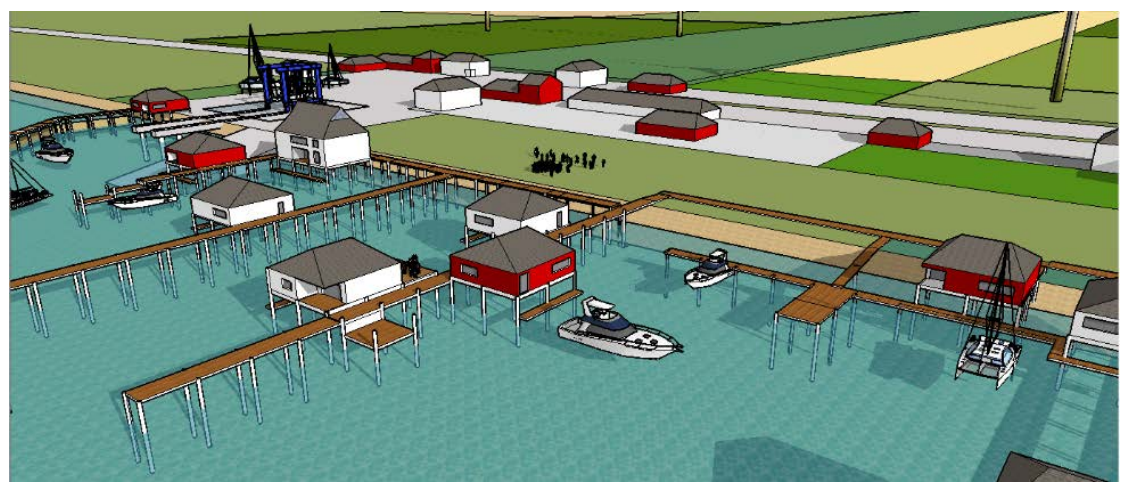

Figure 2. Habitat sur pilotis sur plan d'eau (@ Beynet, 2019).

Mais une telle solution est difficilement envisageable dans le contexte règlementaire actuel en France, car à ce jour l'affectation du plan d'eau portuaire soit à l'usage direct du public, soit à un service public peut faire obstacle à son ouverture à une urbanisation constituée par l'installation d'habitations flottantes par nature de caractère privatif et pérenne. Lorsque le plan d'eau fait partie d'une propriété privée, que ce soit celle d'un particulier ou d'une personne publique, un tel projet est réalisable si les règles générales et locales d'urbanisme le permettent; il est dès lors soumis à la même règlementation que pour les constructions traditionnelles. Il est tenu compte de la surface de plancher projetée, et c'est donc la notion d'emprise au sol qui s'applique, notion que l'article R420$1 \mathrm{du}$ Code de l'urbanisme définit comme "une projection verticale du volume de la construction, tous débords et surplombs inclus".

\subsection{Habitat sur pilotis implanté en partie sur talus ou berge}

L'habitat sur pilotis, figure 3, peut être mis en œuvre, en zone inondable par exemple, mais à condition que ce soit sur terre (terre-plein exondé) et non pas sur un plan d'eau du DPM ou du DPF comme rappelé plus haut. Ensuite il devrait être possible d'implanter de l'habitat sur pilotis sur le talus d'un tel remblai. Cette solution permet de bénéficier des 


\section{Thème 4 - Ouvrages portuaires, offshore et de plaisance}

avantages techniques de la conception sur pilotis Elle consiste à implanter l'habitation sur pilotis, en partie sur le talus d'une berge naturelle, si le document d'urbanisme local le permet, le quai d'accostage étant construit en console, fixé plus bas sur les pieux, au bon niveau pour permettre les transferts de plaisanciers entre le bateau et le quai.
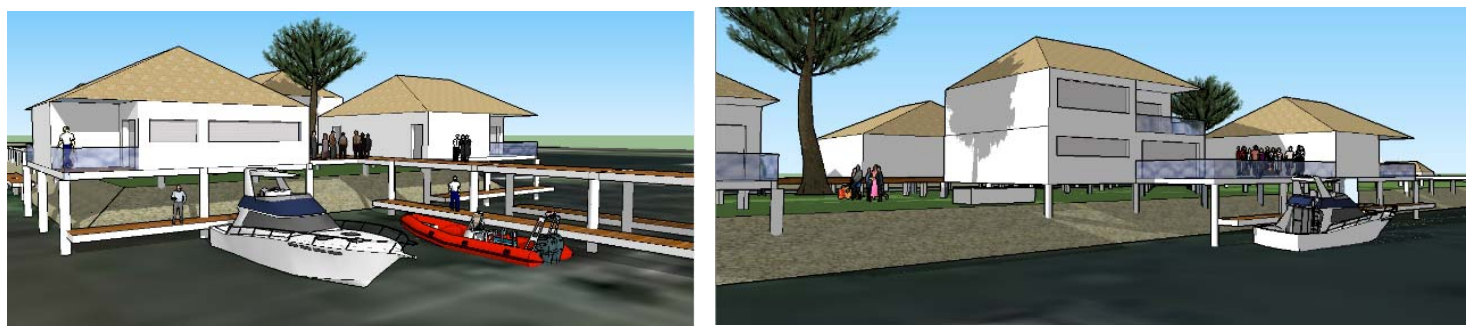

Figure 3. Habitat sur pilotis sur talus ou berges d’un terre-plein. ( Beynet, 2019) Commentaires : Le rez-de-chaussée des habitations est implanté sur le terre-plein ou son talus, fondé sur pilotis et calé au-dessus des plus hautes eaux prévisibles, alors que le quai de débarquement est calé au niveau du franc-bord des bateaux de plaisance et son niveau peut-être surélevé plus tard, pour s'adapter si nécessaire, en fonction de

l'élévation du niveau de l'eau, dans les décennies à venir.

Sur le plan réglementaire, la construction d'habitats sur pilotis pourra être envisagée à condition qu'elle ne soit pas contraire aux dispositions des documents de planification supracommunaux ou communaux. Il faudra ainsi s'assurer de la faisabilité d'un tel projet en considération du zonage du territoire à risque important d'inondation, et bien entendu du PLU et du PPRI.

S'agissant des documents supracommunaux, on citera le schéma directeur d'aménagement et de gestion des eaux, ou encore le schéma d'aménagement et de gestion des eaux, conçu à l'échelle d'une unité hydrographique.

Autre document dont il faut tenir compte : le TRI (territoire à risque important d'inondation. Mis en œuvre en application de la Directive 2007/60/CE du 23/10/2007), on en dénombre 122 en métropole et en outre-mer. Dans ces territoires identifiés par un arrêté du préfet coordonnateur de bassin, des diagnostics permettent de connaître précisément les surfaces inondables et les risques d'inondations.

Il faut s'assurer également de la compatibilité du projet d'installation d'un habitat sur pilotis avec les dispositions du plan de prévention des risques naturels d'inondation, lorsqu'il existe, et qui constitue une servitude d'utilité publique figurant en annexe du plan local d'urbanisme.

L'article L. 321-14 du code de l'environnement sur les Schémas régionaux d'aménagement, de développement durable et d'égalité des territoires (Sraddet), créés en 2015, indique que ce document comporte des dispositions relatives à l'inclusion de la gestion du trait de côte dans les éléments à prendre en compte pour l'élaboration de ces schémas. Ils constituent les documents de planification dans lesquels les enjeux 


\section{XVI'̀mes Journées Nationales Génie Côtier - Génie Civil \\ Le Havre 2020}

environnementaux sont centraux. Leur vocation est d'harmoniser et de mettre en cohérence les politiques d'aménagement du territoire à une échelle pertinente puisque régionale. Ainsi le Sraddet doit-il préciser "les règles générales d'un projet de territoire qui permet d'anticiper et de gérer les évolutions du trait de côte, portant notamment sur les mesures d'amélioration des connaissances, de préservation et de restauration des espaces naturels ainsi que de prévention et d'information des populations. Il détermine les modalités d'un partage équilibré et durable de la ressource sédimentaire".

L'article L321-13 du même code (introduit par la loi n 2016-1087 du 8 août 2016 pour la reconquête de la biodiversité, de la nature et des paysage) dispose d'ailleurs que "Afin d'anticiper l'évolution du trait de côte et de prendre en compte les phénomènes hydrosédimentaires entraînant l'érosion et l'accrétion littorale dans les politiques publiques, l'Etat établit une cartographie fondée sur un indicateur national d'érosion littorale".

Désormais, la logique d'aménagement du littoral prend en considération ce qui ne l'était pas dans le passé : la dynamique du milieu naturel. Toute zone littorale est mobile. Tenant le fait qu'il n'est pas envisageable de protéger toutes les zones aménagées sur le littoral, il sera nécessaire de choisir les espaces à préserver en priorité, ce qui supposera la mise en œuvre de moyens très importants de protection, et de prévoir dans d'autres secteurs des déplacements de population.

La politique nationale de prévention des risques naturels a pour objet de permettre aux populations résidant dans des zones particulièrement exposées de se réinstaller en dehors de ces zones à risques et ainsi d'assurer la mise en sécurité des sites libérés d'une occupation humaine incompatible avec l'existence d'un risque naturel. La règlementation applicable n'est qu'un instrument au service de cette politique. Ainsi en est-il des règles d'utilisation du Fonds de prévention des risques naturels majeurs (FPRNM) dit Fonds Barnier, permettant de recourir en tant que de besoin, soit à l'acquisition amiable. La loi $\mathrm{n}^{\circ}$ 2003-699 du 30 juillet 2003 relative à la prévention des risques technologiques et naturels et à la réparation des dommage a introduit la possibilité de recourir au FPRNM pour financer l'acquisition amiable de biens dont la situation les rendrait éligibles à la procédure d'expropriation, soit à l'expropriation elle-même, après la mise en œuvre d'une procédure de déclaration d'utilité publique, comme le précise aujourd'hui l'article L5611 du code de l'environnement. Dans l'un et l'autre des cas, les biens éligibles sont ceux exposés à des mouvements ou affaissements de terrain des avalanches, des crues torrentielles ou à montée rapide et des submersions marines.

Comme le rappelle le Centre Européen de Prévention du Risque Inondation, dans son guide édité en 2016 et intitulé "Les collectivités territoriales face aux risques littoraux. Élaborer et mettre en œuvre une stratégie de réduction du risque de submersion marine", la Stratégie nationale d'adaptation au changement climatique de 2007 utilise les termes de "recul ou de repli stratégique" pour désigner les opérations conduites par l'État ou les collectivités territoriales visant à supprimer les biens et équipements situés dans les zones 


\section{Thème 4 - Ouvrages portuaires, offshore et de plaisance}

à risques, après évacuation de leurs éventuels occupants. La Stratégie nationale de gestion intégrée du trait de côte de 2012 privilégie pour sa part le terme de "relocalisation des activités et des biens". Le CEPRI observe : "Il ne s'agit pas uniquement de retirer les biens et activités des zones à risque mais également de les réimplanter sur le territoire dans le cadre d'un projet plus global de recomposition de l'espace littoral et arrièrelittoral : Il est nécessaire de planifier maintenant et de préparer les acteurs à la mise en cuvre de la relocalisation à long terme des activités et des biens exposés aux risques littoraux, dans une perspective de recomposition de la frange littorale, et ce même si des mesures transitoires sont mises en œuvre".

Les ministères de la Transition écologique et solidaire, de l'Économie et des Finances et de l'Intérieur ont édité en mars 2019 un guide relatif à la mobilisation du Fonds de prévention des risques naturels majeurs (FPRNM). Le risque "submersion marine" est l'un des aléas concernés. On en retiendra que les études, travaux ou équipements de prévention ou de protection des collectivités territoriales (ETECT) sont éligibles au fonds Barnier, et que cette mesure qui bénéficie aux collectivités territoriales a pour vocation première d'améliorer la sécurité des personnes face aux risques naturels et de réduire les dommages aux biens assurés.

\section{Ouvrages de protection extérieure contre la houle}

Pour les sites peu exposés aux houles longues du large, les ouvrages de protection extérieure peuvent être réalisés selon la conception d'une estacade fixe sur pieux, supportant un voile mince de retombée, figure 4, (justifié par exemple selon les formules de WIEGEL (1960)). Un tel ouvrage est efficace pour atténuer des clapots de périodes courtes, du type mer du vent ou encore les vagues d'étrave générées par des bateaux naviguant devant la marina. La hauteur du voile de retombée peut être augmentée en cas d'élévation du niveau des mers plus rapide que prévu au moment de la conception, de manière à ce que le coefficient de transmission Ct entre la houle incidente et la houle résiduelle admissible reste dans les normes AIPCN pour les bateaux amarrés aux quais. Cette conception sur pieux est en effet plus facilement adaptable que la solution d'une digue à talus avec carapace de protection, car la surélévation nécessiterait de reprendre la carapace du talus extérieur et augmenterait également l'emprise au fond. Cela conduirait à des travaux nettement plus onéreux et peut-être même à des impossibilités en site maritime, en cas de présence de posidonies (protégées par Directives Européennes).
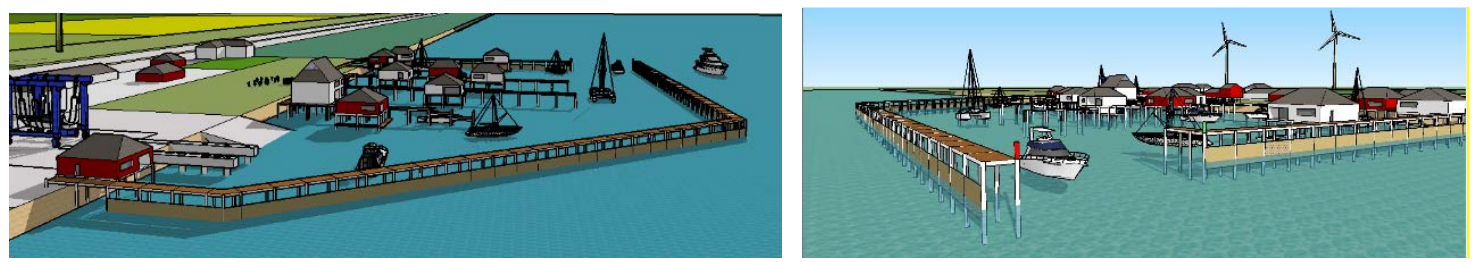

Figure 4. Ouvrage de protection avec voile mince de retombée (@ Beynet, 2019). 


\section{XVI èmes Journées Nationales Génie Côtier - Génie Civil \\ Le Havre 2020}

\section{Environnement littoral, assainissement autonome et énergies renouvelables}

La marina du futur ne devra pas apporter de nuisances à l'environnement. A titre d'exemple, pour compenser les ombres portées sur les fonds (qui appauvrissent la faune et la flore aquatique), des dispositifs comprenant un capteur-concentrateur de lumière du jour en toiture et une transmission de la lumière par fibres optiques en sous face de l'habitation pourraient être mis en œuvre. Par ailleurs, les marinas du futur devront autant que possible être autonomes, aussi bien pour l'assainissement que pour l'énergie.

Il est possible de prévoir une petite station d'épuration individuelle pour ce type d'habitat. De plus, la production d'énergie électrique à partir de l'éolien ou de panneaux photovoltaïques est à maturité à présent. Enfin, les progrès récents dans la production et le stockage d'hydrogène et les piles à combustible permettent de stocker cette énergie.

\section{Conclusions}

Pour tenir compte de l'élévation du niveau des mers dans les décennies à venir, l'habitat littoral devra forcément s'adapter pour rester "hors d'atteinte de la mer". Des solutions techniques existent, elles ont d'ailleurs déjà été mise en œuvre dans d'autres pays en Europe et dans le monde. Mais en France, le contexte réglementaire est très contraignant et il ne permet pas à ce jour l'adaptation évolutive qui serait souhaitable.

\section{Références bibliographiques}

GIEC -OPPENHEIMER M., GLAVOVIC B.C., HINKEL J., VAN DE WAL R., MAGNAN A.K., ABD-ELGAWAD A., CAI R., CIFUENTES-JARA M., DE CONTO R.M., GHOSH T., HAY J., ISLA F., MARZEION B., MEYSSIGNAC B., SEBESVARI Z.- (2019). Sea level rise and implications for low lying islands, Coasts and Communities, In: IPCC Special Report on the Ocean and Cryosphere in a Changing Climate, Chapter 4, $51^{\text {st }}$ Session held on 20-28 September 2019, pp 321-445.

WIEGEL R.L. (1960). Transmission of waves past a rigid vertical thin barrier, Journal of the Waterways and Harbors Division, ASCE, Vol. 86, Issue 1, pp 1-12. 
Thème 4 - Ouvrages portuaires, offshore et de plaisance 